\title{
La Cartografía Social como Herramienta Educativa
}

\begin{tabular}{|c|c|}
\hline & $\begin{array}{r}\text { Autor: César Enrique López Arrillaga } \\
\text { Universidad Latinoamericana y el Caribe, ULAC } \\
\frac{\text { prof.cesarlopez@gmail.com }}{\text { Caracas, Venezuela }}\end{array}$ \\
\hline & $\begin{array}{l}\text { Resumen } \\
\text { El presente ensayo abordará teóricamente la cartografía social } \\
\text { aplicable en el ámbito educativa como herramienta metodológica en los } \\
\text { actores de los procesos enseñanza - aprendizajes en las escuela, con el } \\
\text { objetivo de acercar la escuela a su ámbito comunitario, además relacionar los } \\
\text { aportes que ofrece la cartografía social al ámbito educativo y su definición } \\
\text { planteada por (Diez, Escudero, Carballeda, Barberena, Hallak, Rocha, } \\
\text { Massera, Vázquez, Barceló, Coñuecar, Gómez, Gómez, Feü, Martínez y } \\
\text { Moreno, 2012), además los postulados teóricos presentados por (Herrera, } \\
\text { 2008), planteando las ventajas y desventajas de su aplicación, así mismo la } \\
\text { aplicación de la Cartografía Social en el quehacer educativo, finalmente la } \\
\text { importancia de su realización en el sector educativo que permita a los actores } \\
\text { educativos vincularse con su comunidad, en la búsqueda de soluciones } \\
\text { factibles de los problemas comunes, y la construcción del conocimiento } \\
\text { colectivo. }\end{array}$ \\
\hline
\end{tabular}

Palabras clave: cartografía; educación; docente; escuela. 


\title{
The Social Cartography as an Educational Tool
}

\begin{abstract}
The present essay will theoretically address the social cartography applicable in the educational field as a methodological tool in the actors of the teaching - learning processes in schools, with the aim of bringing the school closer to its community level, in addition to relating the contributions offered by social cartography to the educational field and its definition proposed by (Diez, Escudero, Carballeda, Barberena, Hallak, Rocha, Massera, Vázquez, Barceló, Coñuecar, Gómez, Gómez, Feü, Martínez and Moreno, 2012), as well as the theoretical postulates presented by (Herrera , 2008), proposing the advantages and disadvantages of its application, likewise the application of Social Cartography in the educational task, finally the importance of its realization in the educational sector that allows educational actors to link with their community, in the search of feasible solutions to common problems, and the construction of collective knowledge.
\end{abstract}

Keywords: cartography; education; teacher; school. 


\section{Introducción}

Las herramientas educativas en el desarrollo del siglo XXI, y producto del proceso de globalización y en la era de la postmodernidad hacen imperativo que los docentes se actualicen en su práctica educativa con el objetivo de motivar a sus educandos en un proceso de enseñanza-aprendizaje motivador, dinámico y armónico.

Por tanto, en la brusquedad de las diversas y variadas herramientas consideradas en el ámbito educativo, en el presente se desarrollará la Cartografía Social en su aplicación a la práctica educativa de los docentes como opción de innovación y actualización de su labor, mejorar el ambiente de los estudiantes y su formación integral.

Por otro lado, Diez, Escudero, Carballeda, Barberena, Hallak, Rocha, Massera, Vázquez, Barceló, Coñuecar, Gómez, Gómez, Feü, Martínez y Moreno (2012), señalan que las Cartografías Sociales:

se presentan como una nueva oportunidad de aplicación de formas de conocimiento y posibilidades de intervención social a nivel local. Reconocen desde su práctica una nueva forma de viajar a territorios inexplorados o poco conocidos, llegar ahí donde las significaciones cambian de forma o se tornan inestables, casi como un medio de transporte que nos lleva a los complejos laberintos de los discursos, las representaciones, las historias relatadas y no contadas (pág. 5).

De acuerdo con lo anterior, el autor plantea la Cartografía Social como una opción para la ejecución de nuevas prácticas en la intervención social que se puede alinear al ámbito educativo con el propósito que los estudiantes tengan contacto con lo social y local de su contexto territorial e identificar sus significaciones de todos los elementos que lo representan.

Así mismo, la cartografía interactúa de forma permanente, continua y constante en el entorno donde se aplica, y permite reconocer, identificar y establecer todos los elementos que forman el territorio donde se desenvuelven 
los estudiantes en la construcción colectiva de su ambiente, desde la práctica del mapeo.

Finalmente, el presente ensayo examinará los fundamentos conceptuales de la Cartografía Social, haciendo énfasis en los conceptos y características fundamentales, así mismo se reseñará la metodología de la Cartografía Social, Cartografía Social y ámbito educativo; destacando la relación de la herramienta con la educación, y por último la importancia de la cartografía social en la educación considerando la utilidad de la cartografía social en el proceso de enseñanza-aprendizajes de los estudiantes y la praxis educativa del docente.

\section{Desarrollo}

\subsection{La Cartografía Social}

Para Fals Borda (1987), citado por Vélez, Rátiva y Varela (2012), plantea que:

Se entiende la cartografía social como una metodología participativa y colaborativa de investigación que invita a la reflexión, organización y acción alrededor de un espacio físico y social específico. Como metodología de trabajo en campo y como herramienta de investigación, se concibe a la cartografía social como una técnica dialógica (pág. 155).

De acuerdo con lo anterior, la cartografía social es considerada una opción de metodología en la participación y en colaboración con todos los actores que impulsa la reflexión colectiva en el proceso de la investigación educativa, generando el trazado en lo territorial de las potencialidades, necesidades y otros indicadores de interés para la comunidad educativa.

Por otro lado, Herrera (2008a), plantea que:

la cartografía social es una propuesta conceptual y metodológica que permite construir un conocimiento integral de un territorio, utilizando instrumentos técnicos y vivenciales. Se trata de una herramienta de planificación y transformación 
social, que permite una construcción del conocimiento desde la participación y el compromiso social, posibilitando la transformación del mismo (pág. 3).

En concordancia con el autor, se propone como una herramienta que permite la construcción del conocimiento colectivo de los docentes y estudiantes la institución educativa que permita hallar las posibles vías de soluciones a las problemáticas encontradas en el territorio o contexto social determinado.

Así mismo, Herrera (2008b), señala que:

la cartografía social como instrumento, es un ejercicio participativo que por medio de recorridos, talleres o grupos de discusión, utiliza el mapa como centro de motivación, reflexión y redescubrimiento del territorio en un proceso de conciencia relacional, invitando a los habitantes de un territorio a hablar sobre los mismos y las territorialidades (pág. 3).

En este mismo orden ideas, debe considerarse como un instrumento en el cual los actores sociales podrán expresar libremente todas sus ideas e impresiones de la realidad social-educativa abordada en el ejercicio para ubicar los hallazgos en la comunidad educativa para el descubrimiento de indicadores y situaciones para su estudio, análisis y discusión respectiva.

Según Habegger y Mancila (2006), citados por (Herrera, 2008c), indican que:

por cartografía social como la ciencia que estudia los procedimientos en obtención de datos sobre el trazado del territorio, para su posterior representación técnica y artística, y los mapas, como uno de los sistemas predominantes de comunicación de ésta. A lo largo de los años éstos han ido evolucionando conceptualmente. La dualidad etimológica de la cartografía, con el sufijo que puede significar sin distinción la escritura, la pintura o el dibujo; explora el vínculo entre la grafía (la escritura) y la gráfica (el dibujo), entre los instrumentos de tipo texto y los documentos de tipo imagen (pág. 5). 
En concordancia con lo planteado por el autor, la Cartografía Social permite el manejo de información de una manera dinámica y visual a través del mapeo, recoger las informaciones de forma gráfica ayudando al proceso de investigación igualmente promueve la percepción de las relaciones en el entorno y estudia las consecuencias de las interacciones en lo social y lo territorial, además puede ser considerada como una herramienta de resolución de conflictos, en la cual las partes pueden transformar un problema en diversas soluciones creativas y dinámicas.

Por otra parte, en el ámbito educativo la Cartografía Social permite identificar los elementos y relaciones de los estudiantes-docentes de sus comunidades y la escuela, enlazando dos contextos que deben ser unidos en función de garantizar la calidad educativa del proceso enseñanza-aprendizaje que corresponda con los intereses de formación de los educandos y permita la formación integral y holística.

\subsection{Aplicación de la Cartografía Social}

En la aplicación de la Cartografía Social en las instituciones educativas se considera la participación e integradora de los participantes toman protagonismo en la ejecución y aplicación de la herramienta en el contexto determinado.

Por lo cual, la comunidad educativa juega un papel importante en la investigación, donde los actores sociales aportan sus conocimientos y experiencias de una manera recíproca y de intercambio con el objetivo de la concreción de mapas que permite la actualización de la memoria individual y colectiva de los docentes, estudiantes y demás actores involucrados en el ejercicio.

Así mismo, la acción requiere de la responsabilidad y compromiso de los docentes, estudiantes y demás actores de la escuela, los cuales desde sus perspectivas deben impulsar el ejercicio desde los aportes individuales con la 
finalidad de construir colectivamente el cono miento permitiendo la construcción de la realidad social.

Igualmente, la participación es entendida como las fuerzas individuales y colectivas de todos los actores de la comunidad educativa, en la construcción social del conocimiento, permitiendo el reconocimiento de las debilidades, limitaciones, habilidades y destrezas de la institución educativa.

Por otra parte, la sistematización, debe transcender hacia la organización y practica coherentes de los hallazgos encontrados con la construcción de los conocimientos de manera colectiva, siendo un elemento primordial para encontrar y aprender de la realidad, con el propósito de transformarla con la obtención de los datos, conocimientos y prácticas. Por otra parte, una vez realizado el proceso de aplicación en la metodología planteada y socialización de los hallazgos y la construcción colectiva del conocimiento, en lo siguiente observaremos algunos ejemplos de Cartografía Social aplicada en el ámbito comunitario:

Gráfica 1. Cartografía Social en la comunidad Urbanización Agropecuaria Guaicaipuro, Terrazas 1, 2, 3, 4, 5, 6, 7, 8. Estado Miranda, Venezuela.

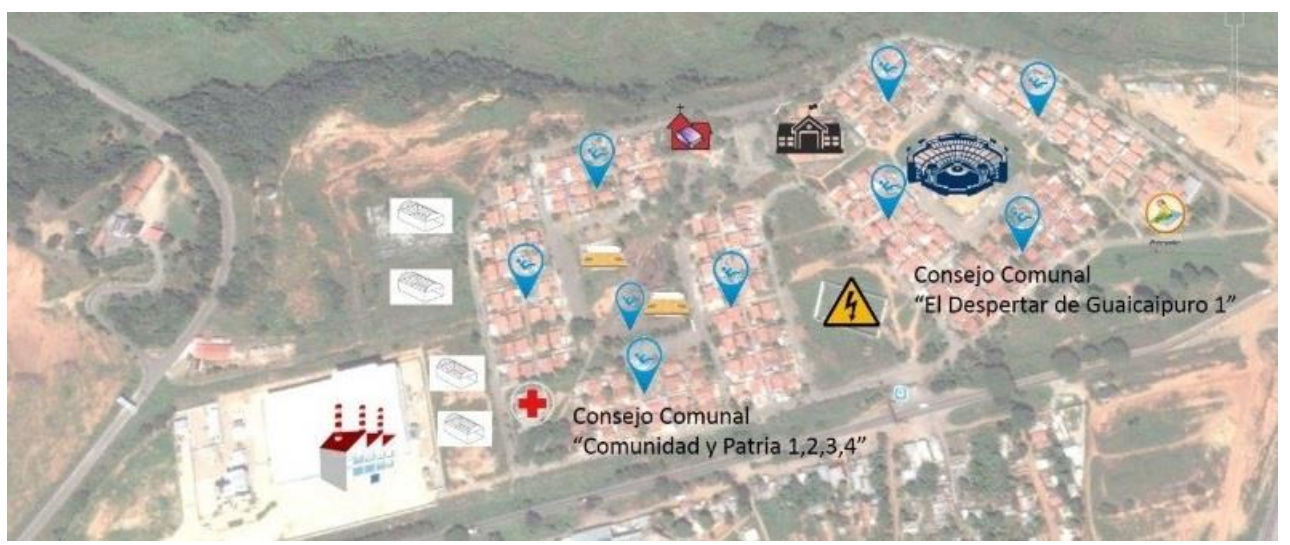

\section{Leyenda:}

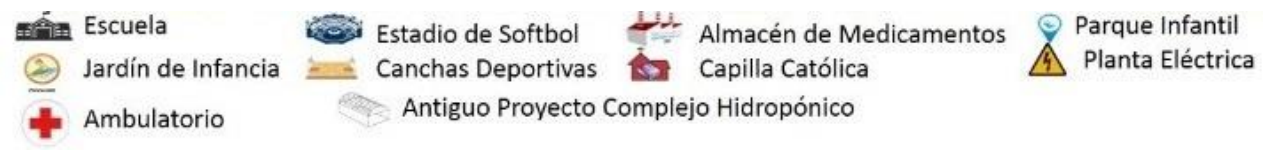

Fuente: El Autor (2018). 
En la gráfica 1, se puede observar la utilidad de la Cartografía Social aplicada en el ámbito comunitario, se hace visible de manera gráfica notable todos los aspectos económicos, sociales, históricos-culturales, seguridad que propician una mejor practica para el abordaje social en el levantamiento de la información requerida para futuros proyectos y soluciones posibles para la comunidad organizada. Fortaleciendo el sentido de pertenencia en la historia local del estudiante con su comunidad, reforzando la identidad de los actores educativos en su contexto geográfico.

\subsection{Cartografía Social y el ámbito educativo}

En el ámbito educativo la cartografía social debe tomarse en cuenta como una herramienta innovadora para su aplicación en la práctica docente, que facilite la vinculación de los estudiantes con su entorno social y comunitaria, que permita la relación Escuela-Comunidad-Familia, fortaleciendo la formación integral y comunitaria, de ciudadanos más receptivos y orientados al trabajo social con sus respectivos espacios comunales. Inculcando el valor de cuidar y proteger su entorno como su hábitat social.

Para Barragán y Amador (2014a): "plantean varias alternativas para la puesta en práctica de la Cartografía Social en el ámbito educativo, que permite llegar a la realidad educativa con varias tonalidades" (pág. 52); y diseñan la siguiente metodología para la educación:

a. Selección del tipo de problemática: en el abordaje de algún problema educativo, debe considerarse todas las condiciones que ocasiono el acontecimiento, los actores del ámbito educativo y las posibles soluciones, igualmente se debe identificar el tipo de problema que se trabajará para dar orientación efectiva de la Cartografía Social.

b. Selección del tipo de mapa: Después de haber identificado los objetivos de la actividad, se debe señalar el tipo de mapa, el cual 
permitirá a los actores intervenir en la problemática seleccionada, además se pueden aplicar diversos tipos de mapa

c. Motivación de los participantes: Los participantes se involucran en la construcción colectiva del conocimiento con la expresión voluntaria y confianza para la comprensión de la problemática para promover planes y soluciones para mejorar $u$ optimizar las realidades.

d. Grupos de trabajo: La organización de grupos con pocos participantes garantiza la efectividad de la actividad, de igual forma debe estructurarse en un moderador quien guiará y fomentará la participación, por otra parte, se designará un sistematizador quien llevará el registro de la actividad.

e. Acuerdo de convenciones: Se debe acordarse el conjunto de signos o símbolos para identificar los indicadores que se utilizarán en el mapa seleccionado, una vez acordado por la mayoría del grupo de trabajo, se inicia la identificación de los mismos.

f. Elaboración del mapa: En esta etapa preparatoria todos los integrantes del grupo participan activamente en la elaboración del mapa, de acuerdo con las características del ámbito territorial de la institución educativo o comunitario.

g. Explicación del mapa: Una vez realizado el mapa, sebe generar la socialización de los grupos de trabajo, para mostrar los resultados y hallazgos de la actividad, se recomienda poseer toda la información sistematizada por escrito, para posteriores análisis e interpretaciones.

h. Acuerdos de trasformación: Los equipos de trabajo deben realizar consideraciones, discusión y consenso para encontrar unificadamente las posibles soluciones, concepciones y conocimientos sobre la problemática planteada al inicio, para obtener soluciones colectivas posibles en la comunidad educativa.

i. Interpretación de los mapas y socialización de la cartografía: Una 
vez culminado el proceso del ejercicio, se debe realizar una lectura reflexiva y critica de la actividad a partir de los resultados, aplicando las diversas técnicas para el análisis de la información, mediada por la retroalimentación de los grupos participantes.

Gráfica 2. Aplicación de la Cartografía Social.

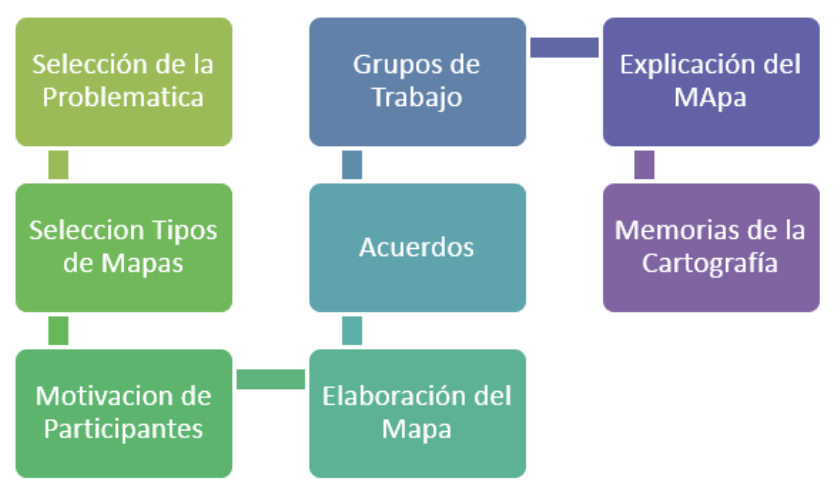

Fuente: Barragán y Amador (2017); adaptación propia (2018).

En este mismo orden de ideas, la metodología para aplicar la cartografía social en el ámbito educativo debe ser flexible y participativa con los docentes, estudiantes y la comunidad, en función de generar espacios colectivos para la creación de conocimiento y aprendizajes, mediante la recolección de información y la socialización de los hallazgos, y crear soluciones compartidas y viables para la transformación social educativa del entorno de los actores participantes, como se puede observar en la gráfica 2, Aplicación de la Cartografía Social.

\subsection{Aportes de la Cartografía Social a la educación}

Para Manzon (2011), citado por Gorostiaga (2017), considera que:

La aparición de la Cartografía Social generó no poca controversia dentro del campo de la educación, en particular por su asociación a un giro posmodernista. Una de las 
principales críticas es que el énfasis puesto en reflejar la multiplicidad y la diferencia puede implicar una mirada relativista (pág. 205).

Por lo tanto, la herramienta en el ámbito educativo permite desarrollar estrategias de identificación de todos los indicadores de los actores que están insertado en el ámbito territorial de la institución educativa, a través del mapeo social con el fin que los estudiantes y docentes realicen un reconocimiento de potencialidades y focos de necesidades, debilidades para impulsar estrategias de intervención educativa y social.

Por otro lado, Andrade (2009a), explica que:

la dimensión educativa de la cartografía social es un trabajo de sistematización, desde una perspectiva de la interpretación de los relatos de algunos de los actores vinculados a la experiencia de la construcción y puesta en práctica de esta metodología (pág. 15).

Considerando lo planteado por el autor, señala que el planteamiento de que la cartografía social es en esencia una experiencia educativa parte de que el objetivo central de la metodología es la construcción colectiva de conocimiento; que se concreta mediante la construcción colectiva de mapas a través de la puesta en juego de diferentes tipos de saberes.

De acuerdo con Beillerot (1988), citado por Andrade (2009b): la cartografía social, se sustenta en la consideración de que los seres humanos cuentan con unos saberes los cuales "son producidos en un contexto histórico y social; hacen referencia a culturas, expresan, muestran modos de socialización y apropiación" (pág. 27).

Por ello, Barragán y Amador (2014b), afirman que:

la cartografía social- pedagógica es una metodología que permite caracterizar e interpretar la realidad comunitariaeducativa de un grupo humano, que se fundamenta en la participación, la reflexión y el compromiso de los agentes sociales implicados (pág. 89). 
De acuerdo con los autores, la cartografía representa una metodología pedagógica para la interpretación de la reubicada educativa que permite que los actores de la escuela identifiquen mediante el mapeo todos los indicadores deseados, mediante la participación y reflexión colectiva.

Así mismo, en el ámbito educativo se debe identificar los actores en su elaboración, construcción y socialización, que se observa en la gráfica $\mathbf{3}$, Actores de la Cartografía Social en Educación.

Gráfica 3. Actores de la Cartografía Social en Educación.

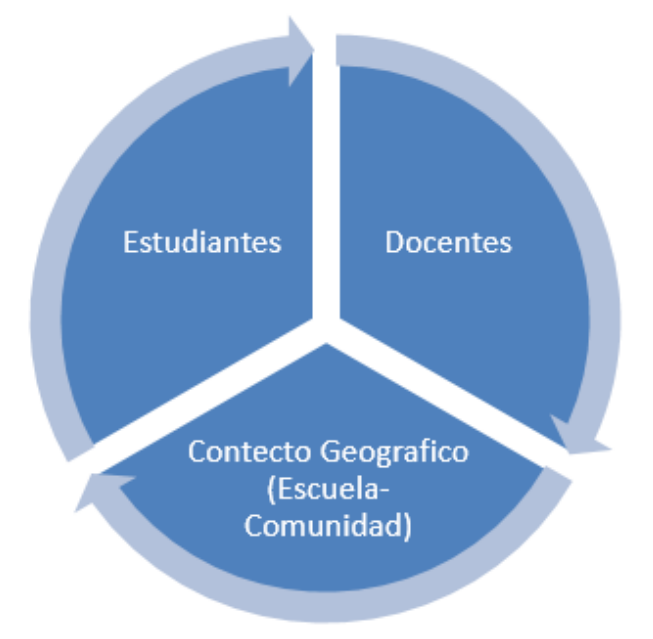

Fuente: El Autor (2018).

En este mismo orden de ideas, en la gráfica 3, se pueden observan los actores involucrados en el montaje, ejecución y socialización de la Cartografía Social en el ámbito educativo, Estudiantes participan directamente en los aportes de ideas, opiniones y presentación de indicadores, zonas, potencialidades y debilidades de su ámbito comunitario y educativo. Docentes su participación radica como mediador y facilitador del proceso de elaboración brindando a los estudiantes las herramientas necesarias para la construcción colectiva del conocimiento en cuanto a sui contexto comunitario-educativo, y Contexto Geográfico es el objeto principal en la aplicación de la Cartografía 
Social, porque en función de este, se propicia toda la construcción, discusión y presentación del mapa con todos sus indicadores y temas a identificar y procesar.

Es importante resaltar, que los docentes deben ser mediadores por excelencia en la motivación de sus estudiantes a la práctica de reconocer su entorno y contexto educativo y comunitario como parte de su identidad local, en función de propiciar el sentido de pertenencia a sus ambientes donde se desenvuelve, dado quien conoce y aprecia, cuida sus espacios y respeta cada elemento que compone su medio social.

\section{Conclusiones}

La complejidad actual de la educación obliga a los docentes a construir y adquirir nuevas herramientas para garantizar cumplir con los fines y objetivos del sistema educativo, que permita la formación integral de los estudiantes, por ello la cartografía social como nueva herramienta educativa, que permite la identificación de elementos en el territorio de las escuelas.

Igualmente, permite la construcción del conocimiento colectivo, el sistema de mapeo genera una participación de la comunidad educativa en la construcción del conocimiento, el cual se desarrolla en un dialogo constantes entre los actores del hecho educativo, que promueve la aceración su comunidad y ámbito territorial.

Por otra parte, se requiere una educación que estimule a los estudiantes por reconocer y analizar sus entornos con el objetivo de impulsar las transformaciones sociales que requieren las comunidades, solo con la formación de ciudadanos identificados con sus contextos sociales, tendremos las herramientas para transformar todos los procesos sociales.

De este modo, la educación tiene una gran deuda histórica con los retos y desafíos actuales del proceso enseñanza-aprendizaje, en el campo educativo hay las inquietudes permanentes de innovar, transformar e aplicar 
nuestras herramientas y estrategias educativas que permita avanzar en la calidad educativa.

\section{Referencias}

Andrade, L. (2009a,b). Sistematización de la dimensión educativa de la

Cartografía Social. Trabajo Especial de Grado del programa Maestría de Educación, énfasis en Educación Popular y Desarrollo Social., Santiago de Cali, República de Colombia: Universidad del Valle, págs. 52-89. Recuperado de:

http://bibliotecadigital.univalle.edu.co/bitstream/10893/10132/1/74050394836.pdf

Barragán, D., \& Amador, J. (2014). La cartografía social-pedagógica: Una oportunidad para producir conocimiento y re-pensar la educación. Itinerario Educativo, (64), 127-141. Recuperado de:

http://revistas.usb.edu.co/index.php/ltinerario/article/download/1422/12 $\underline{15}$

Diez, J., Escudero, H., Carballeda, A., Barberena, M., Hallak, Z., Rocha, E., Massera, C., Vázquez, A., Barceló, M., Coñuecar, V., Gómez, P., Gómez, D., Feü, C., Martínez, N., \& Moreno, N. (2012). Cartografía Social: La investigación e intervención desde las ciencias sociales y experiencia de aplicación. 1a ed. ISBN: 978-987-21581-8-7. Comodoro Rivadavia: Universitaria de la Patagonia, pág. 5-8. Recuperado de: https://www.margen.org/Libro1.pdf

Gorostiaga, J. (2017). Perspectivismo y Cartografía Social: aportes a la educación comparada. Educação \& Realidade, Porto Alegre, 42(3), 877-898. Recuperado de: https://dx.doi.org/10.1590/2175-623665366 Herrera, J. (2008a,b,c). Cartografía Social. Centro Superior de Ciencias Políticas y Sociales. Departamento de Trabajo Social y Servicios Sociales. España: Universidad de la Laguna, págs. 3-5. [Documento en 
línea]: Recuperado de:

https://juanherrera.files.wordpress.com/2008/01/cartografia-social.pdf

Vélez, I., Rátiva, S., \& Varela, D. (2012). Cartografía social como metodología participativa y colaborativa de investigación en el territorio afrodescendiente de la cuenca alta del río cauca. Cuadernos de Geografía. Revista Colombiana de Geografía, 21(2), 5973. Recuperado de:

https://revistas.unal.edu.co/index.php/rcg/article/view/25774/32863 


\section{César Enrique López Arrillaga}

e-mail: prof.cesarlopez@gmail.com

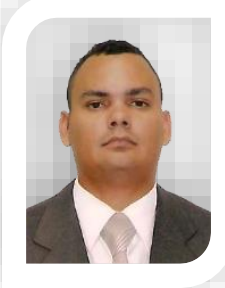

Nací en la Guaira, estado Vargas, Venezuela. Licenciado en Educación de la Universidad Nacional Experimental Simón Rodríguez (UNESR), Magister Scientiarum en Educación Superior de la Universidad Nacional Experimental de la Fuerza Armada (UNEFA), Doctorante del programa Ciencias de la Educación de la Universidad Latinoamericana y del Caribe (ULAC) y estudiante del pregrado de Derecho de la Universidad Bicentenaria de Aragua (UBA), docente de educación primaria de la Unidad Educativa Nacional Bolivariana Guaicaipuro. 